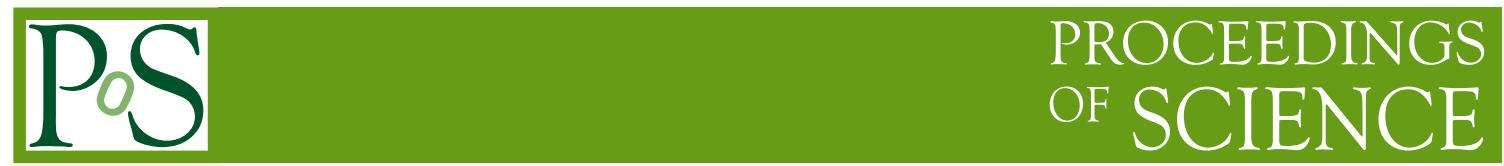

\title{
Rare Beauty and Charm Decays at LHCb
}

\author{
Albert Puig ${ }^{* \dagger}$ \\ École Polytechnique Fédérale de Lausanne \\ E-mail: albert.puig@epfl.ch
}

Flavour changing neutral current decays of heavy flavoured hadrons are suppressed in the Standard Model and can be used to search for new physics contributions. We report a summary of the latest results from the LHCb collaboration on rare decays of beauty and charm hadrons.

2013 Kaon Physics International Conference,

29 April-1 May 2013

University of Michigan, Ann Arbor, Michigan - USA

\footnotetext{
*Speaker.

†n behalf of the LHCb collaboration
} 


\section{Introduction}

Rare decays of $b$ and $d$ hadrons are suppressed or forbidden in the SM and thus constitute a very sensitive environment for the study of NP. Since they mostly occur via loop diagrams, new particles - with masses up to higher scales than those directly accessible - could affect these loops and modify properties of decays, such as branching fractions, angular disttributions of decay products or $C P$ asymmetries. In case of agreement with the SM predictions, the obtained results can be used to set constraints on the possible NP contributions.

The LHCb experiment [1], dedicated to the study of heavy flavor physics at the Large Hadron Collider (LHC), is very well suited for these kind of searches. It profits from the large $b \bar{b}$ and $c \bar{c}$ cross sections at the LHC thanks to highly performing trigger [2] and particle identification [3] systems, which allow, in combination with excellent tracking capabilities, for a precise determination of secondary vertices, impact parameters and momenta.

The latest results obtained for the search of the $B_{(s)} \rightarrow \mu^{+} \mu^{-}, B_{(s)} \rightarrow \mu^{+} \mu^{-} \mu^{+} \mu^{-}, B^{+} \rightarrow$ $\pi^{+} \mu^{+} \mu^{-}$and $D_{(s)}^{+} \rightarrow \pi^{ \pm} \mu^{+} \mu^{\mp}$ channels, as well as the measurement of the properties of $B^{0} \rightarrow$ $K^{* 0} \mu^{+} \mu^{-}$and $B^{0} \rightarrow K^{* 0} e^{-} e^{+}$, are presented here. These analyses rely on $1 \mathrm{fb}^{-1}$ of $p p$ collisions collected by the LHCb experiment in 2011 at a center-of-mass energy of $\sqrt{s}=7 \mathrm{TeV}$. The $B_{(s)} \rightarrow \mu \mu$ analysis adds, on top of these data, $1.1 \mathrm{fb}^{-1}$ of $p p$ collisions in 2012 at a center-of-mass energy of $\sqrt{s}=8 \mathrm{TeV}$.

The presented analyses, while different due to the different nature of the studied channels, have several common general features:

- Background from random combinations of tracks, the so-called combinatorial background, is suppressed using multivariate techniques based on the kinematical properties of the decays.

- Specific backgrounds that peak in the signal region are reduced by making use of dedicated rejection criteria.

- Control channels are used wherever possible to develop selection criteria, and to assess signal and background shapes. The aim in all cases is to reduce, as much as possible, the reliance on simulation.

- Branching fractions are measured by normalizing to control channels using the formula

$$
\mathscr{B}(\text { signal })=\mathscr{B}(\text { control }) \times \frac{\varepsilon_{\text {control }}}{\varepsilon_{\text {signal }}} \times \frac{f_{\text {control }}}{f_{\text {signal }}} \times \frac{N_{\text {control }}}{N_{\text {signal }}}(\times \kappa),
$$

where $\varepsilon$ corresponds to the selection efficiency, $f$ is the $B$ fragmentation fraction, $N$ is the yield and the $\kappa$ parameter accounts, in some cases, for the $S$-wave contributions to the normalization channel yield. In this way, the uncertainties coming from the imperfect knowledge of the luminosity and the $b \bar{b}$ and $c \bar{c}$ cross sections can be avoided.

\section{Search for $B_{(s)} \rightarrow \mu^{+} \mu^{-}$}

The $B_{(s)} \rightarrow \mu^{+} \mu^{-}$decays are flavor changing neutral currents (FCNC) and therefore are only allowed at loop level within the SM. In addition, they are further suppressed by the the GIM 
mechanism and by their helicity structure. The SM prediction for their ( $C P$ averaged) branching fractions is [4]

$$
\begin{aligned}
\mathscr{B}\left(B_{s}^{0} \rightarrow \mu^{+} \mu^{-}\right)^{C P} & =(3.23 \pm 0.27) \times 10^{-9} \\
\mathscr{B}\left(B^{0} \rightarrow \mu^{+} \mu^{-}\right)^{C P} & =(1.07 \pm 0.10) \times 10^{-10},
\end{aligned}
$$

which, as discussed in [5], has to be corrected for the finite width difference in the $B_{s}^{0}$ system [6]. Therefore, the prediction for the $B_{s}^{0}$ branching fraction within the SM is estimated to be $\mathscr{B}\left(B_{s}^{0} \rightarrow\right.$ $\left.\mu^{+} \mu^{-}\right)=(3.54 \pm 0.03) \times 10^{-9}$. Contributions from new processes or heavy particles, for example in the context of MSSM at large $\tan \beta$, could significantly enhance these branching fractions.

This analysis is performed on the $1.0 \mathrm{fb}^{-1}$ data sample collected in 2011 at $7 \mathrm{TeV}$ center of mass energy combined with an additional $1.1 \mathrm{fb}^{-1}$ of data collected at $\sqrt{s}=8 \mathrm{TeV}$ in 2012 [7]. Candidates passing a soft preselection are classified according to their dimuon mass and the output of a multivariate (Boosted Decision Tree, BDT) discriminant built from topological and kinematical variables and trained with $b \bar{b} \rightarrow \mu^{+} \mu^{-} X$, where $X$ is any set of particles, as background and MC simulation for the signal.

The analysis strategy consists in deriving the expected number of background and signal events in each bin for a given $\mathscr{B}$ hypothesis and then to compare these numbers with the observed ones making use of the $C L_{s}$ method [8], which provides a measure of the compatibility of the observed distribution with the signal plus background and background-only hypotheses. An inclusive $B^{0} \rightarrow h^{+} h^{-}$sample, selected as the signal except for the particle identification cuts, is used as control channel for the extraction of the signal BDT and mass distributions from data. The combinatorial background distribution is determined from the mass sidebands by interpolating into the mass region. The peaking background BDT shape is assumed to be the same as the signal one, while its mass distribution is determined from simulation. In the extraction of the branching ratio, two normalization channels, $B^{+} \rightarrow J / \psi K^{+}$and the exclusive $B^{0} \rightarrow K^{+} \pi^{-}$, are averaged.

A simultaneous unbinned maximum likelihood fit to the mass projections in the BDT bins is performed to extract the signal distribution. The signal mass region is then divided in bins, and in each bin of mass and BDT output the number of expected and observed yields is compared. The comparison yields that the probability that the background processes can produce the observed number of $B_{s}^{0} \rightarrow \mu^{+} \mu^{-}$, obtained by the $C L_{s}$ method (Fig. 1) is $5 \times 10^{-4}$, which corresponds to a statistical significance of $3.5 \sigma$. This is the first evidence for the $B_{s}^{0} \rightarrow \mu^{+} \mu^{-}$decay, which is observed a branching fraction

$$
\mathscr{B}\left(B_{s}^{0} \rightarrow \mu^{+} \mu^{-}\right)=\left(3.2_{-1.2}^{+1.5}\right) \times 10^{-9},
$$

consistent with the SM expectation. In the case of $B^{0} \rightarrow \mu^{+} \mu^{-}$, the data in the signal mass window are consistent with the background expectation and the world's best upper limit is set (Fig. 1) at

$$
\mathscr{B}\left(B^{0} \rightarrow \mu^{+} \mu^{-}\right)<9.4 \times 10^{-10} \text { at } 95 \% \text { C.L. }
$$

\section{Search for $B_{(s)} \rightarrow \mu^{+} \mu^{-} \mu^{+} \mu^{-}$}

The $B_{(s)} \rightarrow \mu^{+} \mu^{-} \mu^{+} \mu^{-}$decays are strongly suppressed in the SM. They contain two contrbutions: a resonant one, in which two opposite-charged muons come from a $J / \psi$ and the other two from 

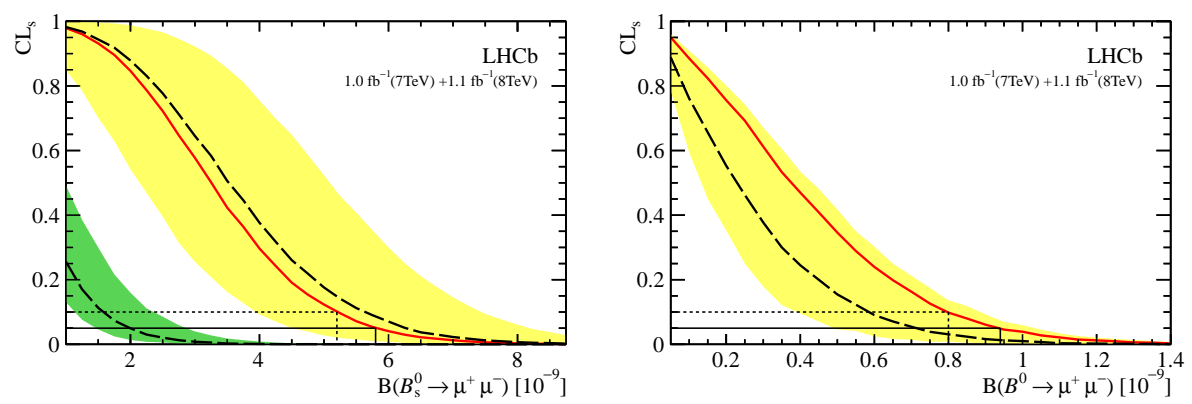

Figure 1: $C L_{s}$ as a function of the assumed $\mathscr{B}$ for $B_{s}^{0} \rightarrow \mu^{+} \mu^{-}$(left) and $B^{0} \rightarrow \mu^{+} \mu^{-}$(right) decays for the combined $2011+2012$ dataset. The long dashed gray curves are the medians of the expected $C L_{S}$ distributions if background and SM signal were observed, with the yellow area covering $34 \%$ of the expected $C L_{S}$ on each side. The solid red curves are the observed $C L_{S}$. For the left plot, the long dashed grey curve in the green area is the expected $C L_{s}$ distribution in the background-only hypothesis, with the filled area covering $34 \%$ of the expected $C L_{s}$ distribution on each side of the line.

a $\phi$ (Fig. 2a), with a branching fraction of $(2.3 \pm 0.9) \times 10^{-8}$ in the SM [9], and a non-resonant one (Fig. 2b), expected to have a branching fraction lower than $10^{-10}$ [10]. These branching fractions can be enhanced in the MSSM via the production of a scalar $S$ and pseudoscalar $P$ sgoldstinos, each decaying into a muon pair (Fig. 2c); this makes the $B_{(s)} \rightarrow \mu^{+} \mu^{-} \mu^{+} \mu^{-}$decay particularly sensitive to NP scenarios.

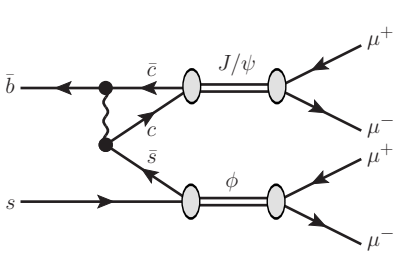

(a) Resonant component.

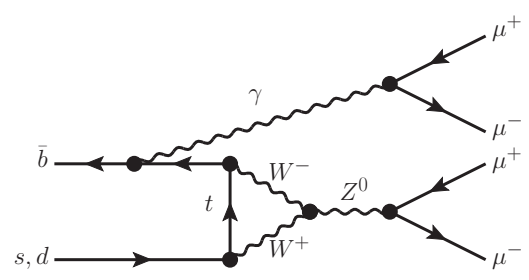

(b) Non resonant component.

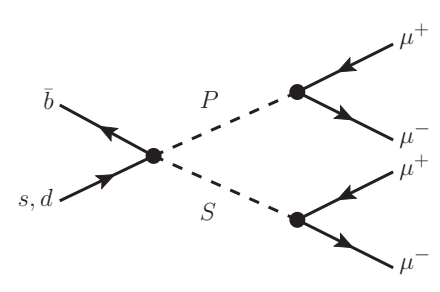

(c) Supersymmetric $B \rightarrow P S$.

Figure 2: Feynman diagrams for (a) resonant and (b) non resonant $B_{(s)} \rightarrow \mu^{+} \mu^{-} \mu^{+} \mu^{-}$decays in the SM, as well as in the (c) MSSM via the intermediate $B \rightarrow P S$ decay.

In this analysis [11], the resonant $B_{s}^{0} \rightarrow J / \psi \phi$ component is removed and used as a control sample for the development of the selection criteria. In addition, the decay $B^{0} \rightarrow J / \psi K^{* 0}$, with $J / \psi \rightarrow \mu^{+} \mu^{-}$and $K^{* 0} \rightarrow K^{+} \pi^{-}$, is used as a normalization channel for the extraction of the $B_{(s)} \rightarrow \mu^{+} \mu^{-} \mu^{+} \mu^{-}$branching fractions.

Both the signal and the control channel selection criteria are based on particle identification, separation between the $B$ vertex and the primary vertex and the quality of the $B$ decay vertex. Cut values are kept the same in both cases, except for the particle identification requirements on the daughters of the $K^{* 0}$.

Sources of peaking background to $B_{(s)} \rightarrow \mu^{+} \mu^{-} \mu^{+} \mu^{-}$, such as $B^{0} \rightarrow \psi(2 S)\left(\rightarrow \mu^{+} \mu^{-}\right) K^{* 0}(\rightarrow$ $\left.K^{+} \pi^{-}\right)$, have been studied and found to be negligible. Therefore, the only considered background in the signal fit is that coming from random combinations of muons, which is estimated from the 
mass sidebands, where no signal is expected. No evidence of signal is found, so upper limits are set for the non-resonant component within the SM [11]

$$
\begin{array}{ll}
\mathscr{B}\left(B_{s}^{0} \rightarrow \mu^{+} \mu^{-} \mu^{+} \mu^{-}\right)<1.2(1.6) \times 10^{-8} & \text { at } 90 \%(95 \%) \text { C.L. } \\
\mathscr{B}\left(B^{0} \rightarrow \mu^{+} \mu^{-} \mu^{+} \mu^{-}\right)<5.3(6.6) \times 10^{-9} & \text { at } 90 \% \text { (95\%) C.L. }
\end{array}
$$

and for the MSSM, assuming the masses of the sgoldstinos to be $m_{S}=2.5 \mathrm{GeV} / c^{2}$ and $m_{P}=$ $214 \mathrm{MeV} / c^{2}$ :

$$
\begin{aligned}
& \mathscr{B}\left(B_{s}^{0} \rightarrow S\left(\rightarrow \mu^{+} \mu^{-}\right) P\left(\rightarrow \mu^{+} \mu^{-}\right)\right)<1.2(1.6) \times 10^{-8} \quad \text { at } 90 \% \text { (95\%) C.L. }, \\
& \mathscr{B}\left(B^{0} \rightarrow S\left(\rightarrow \mu^{+} \mu^{-}\right) P\left(\rightarrow \mu^{+} \mu^{-}\right)\right)<5.1(6.6) \times 10^{-9} \quad \text { at } 90 \% \text { (95\%) C.L. }
\end{aligned}
$$

\section{Study of $B^{0} \rightarrow K^{* 0} \mu^{+} \mu^{-}$}

The $b \rightarrow s(d) l^{+} l^{-}$FCNC transitions are forbidden at tree level in the SM and can only happen in loops, either through penguin or box diagrams. Possible NP effects could arise in these loops from right-handed currents or new operators, resulting in large deviations from SM predictions.

The $B^{0} \rightarrow K^{* 0} \mu^{+} \mu^{-}$decay is very rich in observables, which can help in constraining the size of NP. Its helicity structure can be tested through the distributions of three angles - the helicity angle of the muon, $\theta_{l}$, between the $\mu^{+}\left(\mu^{-}\right)$in the dimuon rest frame and the dimuon in the $B^{0}\left(\bar{B}^{0}\right)$ rest frame, between the kaon in the $K^{* 0}$ rest frame and the $K^{* 0}$ in the $B^{0}$ rest frame, the helicity angle of the kaon, $\theta_{K}$, between the kaon in the $K^{* 0}$ rest frame and the $K^{* 0}$ in the $B^{0}$ rest frame, and the angle $\phi$ between the decay planes of the dimuon and the $K^{* 0}$ systems in the $B^{0}$ rest frametogether with $q^{2}$, which completely describe the kinematics of the decay.

In the analyses reported here $[12,13]$, the $B^{0} \rightarrow K^{* 0} \mu^{+} \mu^{-}$differential branching fraction, angular observables and $A_{C P}$ are measured in bins of $q^{2}$. For the normalization of the branching fraction, shown in Fig. 3, as well as for the extraction of the production and detection asymmetries for $A_{C P}$, the $B^{0} \rightarrow K^{* 0} J / \psi$ channel is used.

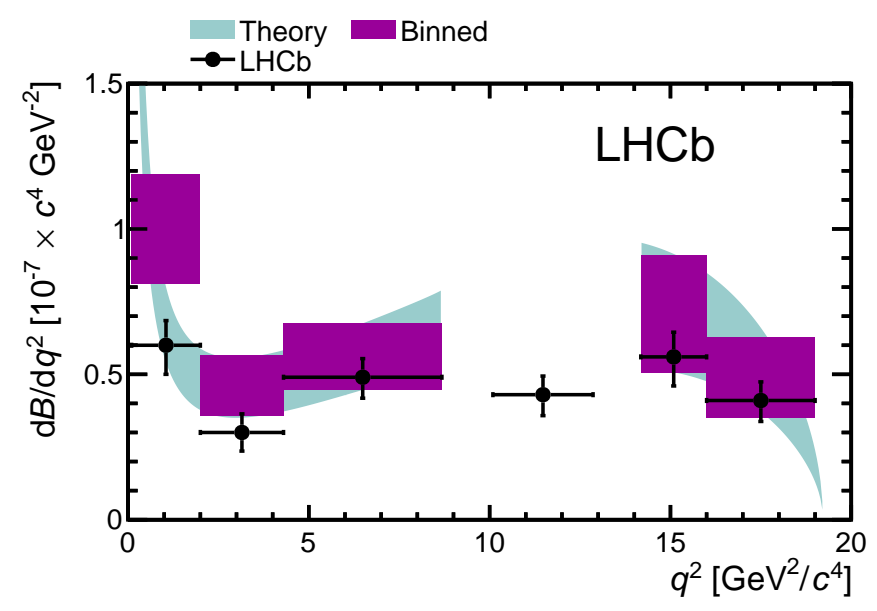

Figure 3: Differential branching fraction of $B^{0} \rightarrow K^{* 0} \mu^{+} \mu^{-}$as a function of $q^{2}$, along with theory prediction, both binned and unbinned. Points include statistical and systematic uncertainties. 
The full $B^{0} \rightarrow K^{* 0} \mu^{+} \mu^{-}$differential decay distribution is parametrized by six $q^{2}$ dependent amplitudes. However, due to the low statistics, a symmetry of the system is exploited and a reduced expression for the angular distribution, containing only four observables $\left(A_{\mathrm{FB}}, F_{\mathrm{L}}, S_{9}\right.$ and $\left.S_{3}\right)$, is obtained. The rate averaged values of these observables are obtained by means of an unbinned maximum likelihood fit to the invariant mass and the angular distribution of the candidates, and the results are shown, together with SM predictions [14], in Fig. 4 [13]. These are the most precise measurements of these quantities to date and are consistent with the SM predictions.

Furthermore, in the SM, the forward-backward asymmetry of the dimuon system changes sign at a well-defined point in $q^{2}$, which is largely free from form-factor uncertainties. The first measurement of this zero-crossing point is performed, yielding

$$
q_{0}^{2}=\left(4.9_{-1.3}^{+1.1}\right) \mathrm{GeV}^{2} / c^{4},
$$

to be compared with the SM predictions, in the range $4.0-4.3 \mathrm{GeV}^{2} / c^{4}$.
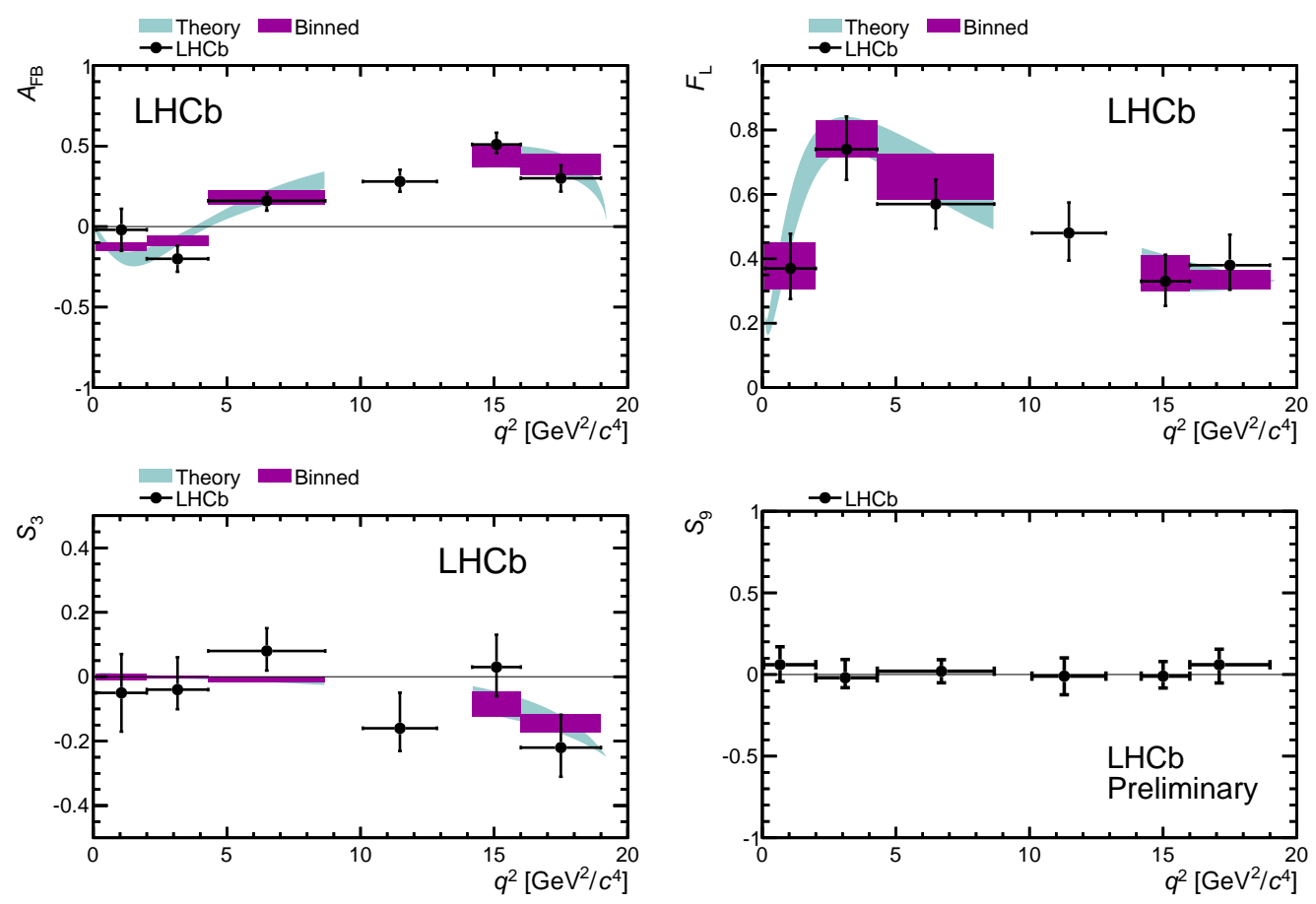

Figure 4: Distribution of (top left) forward-backward asymmetry $\left(A_{\mathrm{FB}}\right)$, (top right) longitudinal polarization of the $K^{* 0}\left(F_{\mathrm{L}}\right.$ ), (bottom left) asymmetry $S_{3}$ and (bottom right) asymmetry $S_{9}$ as a function of $q^{2}$. Theory bands (binned and unbinned) are overlaid in all plots except $S_{9}$, for which the SM predicts a small value of $\mathscr{O}\left(10^{-3}\right)$.

The $C P$ asymmetry of $B^{0} \rightarrow K^{* 0} \mu^{+} \mu^{-}$has also been measured in bins in $q^{2}$, as shown in Fig. 5. Values have been obtained by averaging over magnet polarities and production and detection asymmetries are extracted from $B^{0} \rightarrow K^{* 0} J / \psi$. The result, consistent with the SM prediction within $1.8 \sigma$, is the most precise measurement to date. In addition, $A_{C P}$ is integrated over the full $q^{2}$ range, obtaining

$$
A_{C P}=-0.072 \pm 0.040(\text { stat }) \pm 0.005 \text { (syst) }
$$




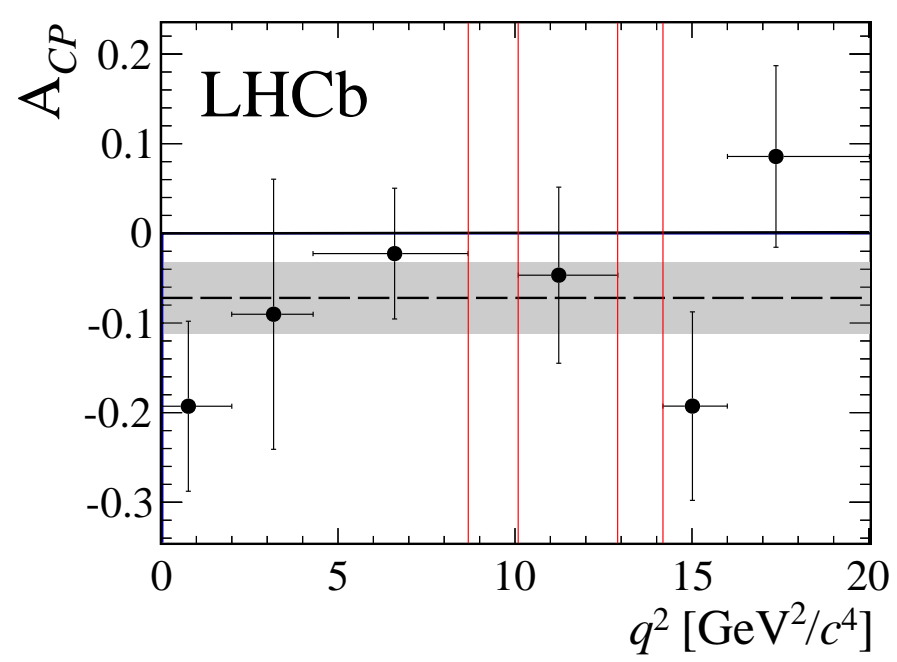

Figure 5: Fitted value of $A_{C P}$ in $B^{0} \rightarrow K^{* 0} \mu^{+} \mu^{-}$in bins of $q^{2}$, including statistical and systematic errors, with the points plotted at the mean value of $q^{2}$ in each bin. The dashed line corresponds to the $q^{2}$-integrated value and the grey band corresponds to its $1 \sigma$ uncertainty.

\section{5. $B^{0} \rightarrow K^{* 0} e^{-} e^{+}$branching fraction at low dilepton mass}

In the SM, the photon helicity is predominantly left-handed, with a small right-handed current arising from long-distance effects and the quark masses. Information on the photon polarization can be extracted from the $B^{0} \rightarrow K^{* 0} l^{+} l^{-}$decays in the low $q^{2}$ region, where the photon contribution is dominant. Due to the lower lepton mass, $B^{0} \rightarrow K^{* 0} e^{+} e^{-}$has a higher sensitivity to photon polarization than $B^{0} \rightarrow K^{* 0} \mu^{+} \mu^{-}$, and is considered complementary to it due to its higher sensitivity to the Wilson coefficient $C_{7}^{\prime}$, compared to $C_{9}^{\prime}$. It suffers, however, from worst resolution due to the sizeable Bremsstrahlung effects.

In the analysis presented here [15], the differential branching fraction of $B^{0} \rightarrow K^{* 0} e^{+} e^{-}$ is studied in the dilepton invariant mass range $30<m_{e^{+} e^{-}}<1000 \mathrm{MeV} / c^{2}$. The chosen range minimizes the contamination from $B^{0} \rightarrow K^{* 0} \gamma$ and avoids multiple scattering effects at low masses, which make angles very difficult to measure. The $B^{0} \rightarrow J / \psi\left(\rightarrow e^{+} e^{-}\right) K^{* 0}$ decay has been used to control the signal shape and as normalization channel.

Radiative $B^{0} \rightarrow K^{* 0} \gamma$ decays with photon conversion are specifically removed with a cut on the conversion vertex, while $B^{0} \rightarrow D^{-}\left(\rightarrow e^{-} v K^{* 0}\right) e^{+} v$ is removed requiring $m_{K^{* 0} e^{-}}>1.9 \mathrm{GeV} / c^{2}$. Remaining backgrounds are combinatorial, $B^{0} \rightarrow K^{* 0} \gamma$ and partially reconstructed $B$ decays.

The data are separed into two samples according to the hardware trigger: events which have triggered on one of the electrons (HWElectron) and events which have triggered on another particle of the event (HWTIS), on the right and left in Fig. 6, respectively. The signal decay has been observed with $4.6 \sigma$ significance with a branching fraction of

$$
\mathscr{B}\left(B^{0} \rightarrow K^{* 0} e^{+} e^{-}\right)^{30-1000 \mathrm{MeV} / c^{2}}=\left(3.1_{-0.8}^{+0.9}{ }_{-0.3}^{+0.2} \pm 0.2\right) \times 10^{-7},
$$

where the systematic uncertainties are well below the statistical ones. Future improvements will include an angular fit, from which the photon polarization can be extracted. 

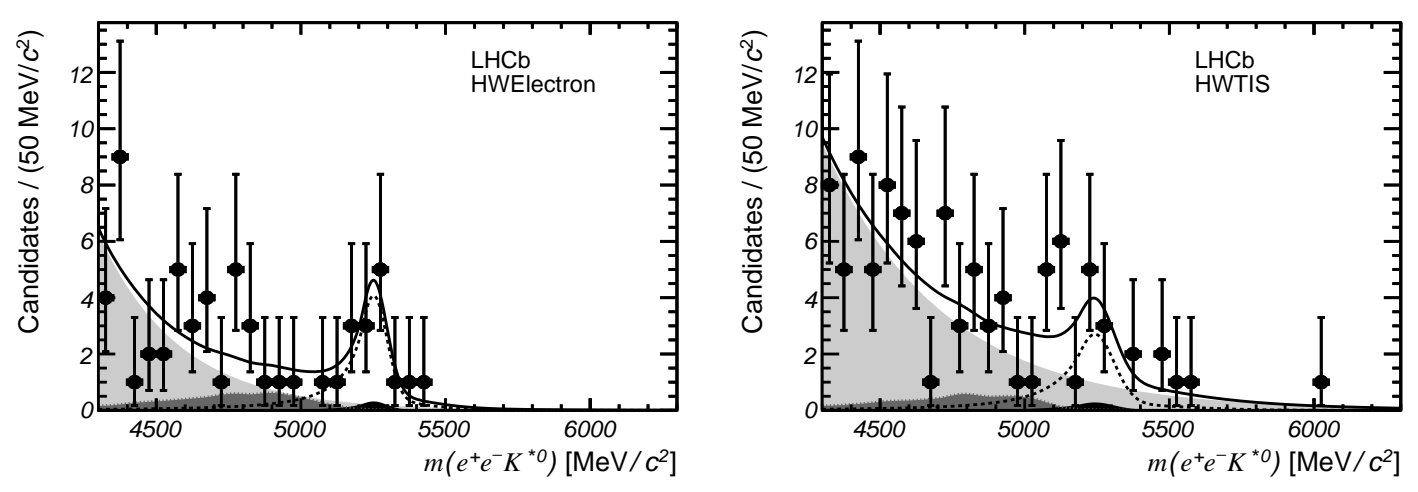

Figure 6: Invariant mass distribution for $B^{0} \rightarrow K^{* 0} e^{+} e^{-}$candidates for the HWElectron (left) and HWTIS (right) trigger categories. The dashed line corresponds to the signal PDF, the light gray area to the combinatorial background, the medium gray area to the partially reconstructed $B$ decays and the black one to $B^{0} \rightarrow K^{* 0} \gamma$.

\section{Search for $B^{+} \rightarrow \pi^{+} \mu^{+} \mu^{-}$}

The $B^{+} \rightarrow \pi^{+} \mu^{+} \mu^{-}$decay is a FCNC $b \rightarrow d l^{+} l^{-}$transition with a SM branching fraction of $\mathscr{B}=(2.0 \pm 0.2) \times 10^{-8}[16]$. No $b \rightarrow d l^{+} l^{-}$transitions have been previously detected, since in the SM they are suppressed relative to $b \rightarrow s l^{+} l^{-}$by $\left|V_{t d}\right|^{2} /\left|V_{t s}\right|^{2}$; this suppression does necessarily apply to models including NP, and $B^{+} \rightarrow \pi^{+} \mu^{+} \mu^{-}$may be more sensitive to the effect of new particles than $B^{+} \rightarrow K^{+} \mu^{+} \mu^{-}$.

These decays provide an alternative way of measuring $\left|V_{t d}\right|^{2} /\left|V_{t s}\right|^{2}$ with respect to radiative decays and mixing processes:

$$
R \equiv \frac{B^{+} \rightarrow \pi^{+} \mu^{+} \mu^{-}}{B^{+} \rightarrow K^{+} \mu^{+} \mu^{-}}=\left(\frac{\left|V_{t d}\right|}{\left|V_{t s}\right|}\right)^{2} f^{2}
$$

where $f$ corresponds to the ratio of relevant form factors and Wilson coefficients integrated over the relevant phase space.

The analysis presented here [17] makes use of $B^{+} \rightarrow K^{+} J / \psi\left(\rightarrow \mu^{+} \mu^{-}\right)$, which is explicitly vetoed, for extracting the signal shape, the misidentified $B \rightarrow K \mu \mu$ shape and also as a normalization channel. After the selection, remaining backgrounds are the combinatorial, partially reconstructed $B$ decays and the peaking $B^{+} \rightarrow K^{+} \mu^{+} \mu^{-}$and $B^{+} \rightarrow \pi^{+} \pi^{-} \pi^{+}$, which arise from particle misidentification.

The $B^{+} \rightarrow \pi^{+} \mu^{+} \mu^{-}, B^{+} \rightarrow K^{+} \mu^{+} \mu^{-}$and $B^{+} \rightarrow J / \psi K^{+}$yields are extracted from a simultaneous fit to the invariant mass distributions of $B^{+} \rightarrow J / \psi K^{+}, B^{+} \rightarrow J / \psi K^{+}$with $K \rightarrow \pi$ mass hypothesis, $B^{+} \rightarrow \pi^{+} \mu^{+} \mu^{-}$and $B^{+} \rightarrow K^{+} \mu^{+} \mu^{-}$. The fitted yield for $B^{+} \rightarrow \pi^{+} \mu^{+} \mu^{-}$, shown in Fig. 7 , corresponds to an observation with $5.2 \sigma$ significance. In addition, a branching fraction of

$$
\mathscr{B}\left(B^{+} \rightarrow \pi^{+} \mu^{+} \mu^{-}\right)=(2.3 \pm 0.6(\text { stat }) \pm 0.1(\text { syst })) \times 10^{-8}
$$

compatible with the SM prediction, is measured. 

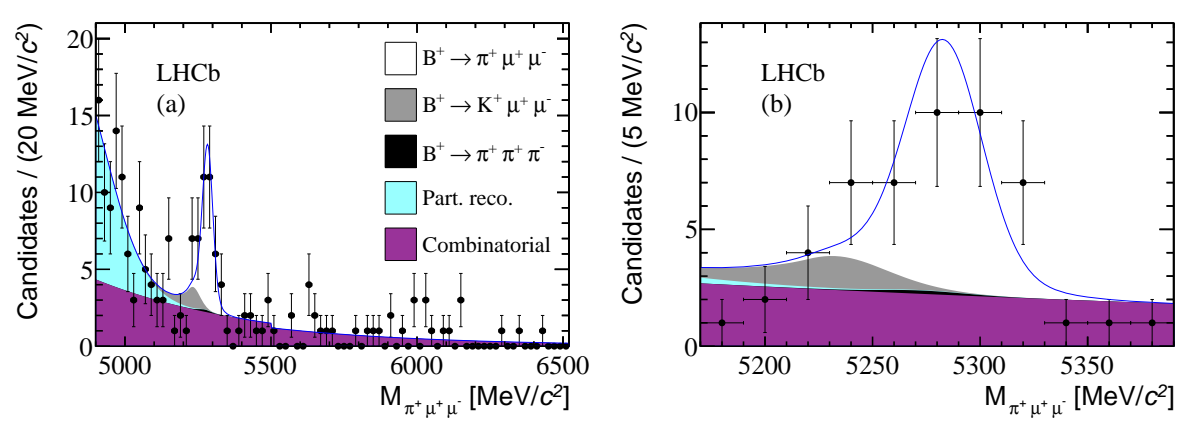

Figure 7: Invariant mass distribution of $B^{+} \rightarrow \pi^{+} \mu^{+} \mu^{-}$candidates with the fit projection overlaid in the full mass range (left) and in the region around the $B$ mass (right).

Taking the measured $B^{+} \rightarrow K^{+} \mu^{+} \mu^{-}$and the relative efficiencies to the signal channel, Eq. 6.1 allows to calculate

$$
\left|V_{t d}\right|^{2} /\left|V_{t s}\right|^{2}=0.266 \pm 0.035 \text { (stat) } \pm 0.003 \text { (syst) }
$$

which is compatible with previous determinations and does not include theoretical uncertainties, e.g., those raising from form factors.

\section{Search for $D_{(s)}^{+} \rightarrow \pi^{ \pm} \mu^{+} \mu^{\mp}$}

FCNC processes in the $D$ meson system, contrary to the case of the $B$ mesons, suffer an almost exact GIM cancellation and therefore are more suppressed, leading to expected branching fractions in the SM of $\mathscr{O}\left(10^{-9}\right)$. NP effects could increase this branching fraction up to an order of magnitude [18].

The presented study [19] searches for non-resonant $D_{(s)}^{+} \rightarrow \pi^{+} \mu^{-} \mu^{+}$decays, making use of the resonant $D_{(s)}^{+} \rightarrow \pi^{-} \phi\left(\rightarrow \mu^{+} \mu^{+}\right)$, with a branching fraction of $\mathscr{O}\left(10^{-6}\right)$, for selection optimization, signal shape extraction and $\mathscr{B}$ normalization. The $\mu^{-} \mu^{+}$invariant mass distribution, shown in Fig. 8, is divided in bins in order to also take into account the contributions of resonant $\omega / \rho \rightarrow \mu^{+} \mu^{-}$.

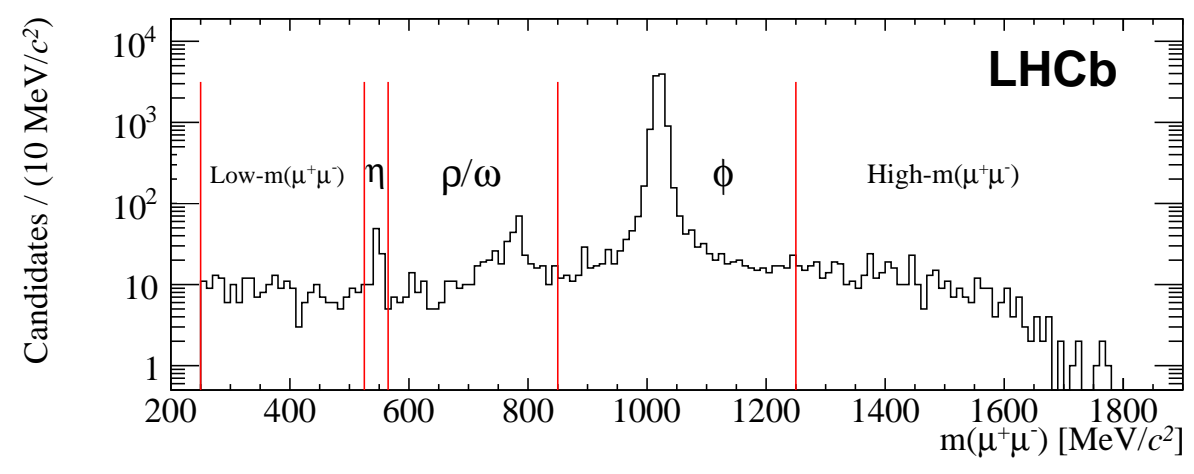

Figure 8: Dimuon spectrum of $D_{(s)}^{+} \rightarrow \pi^{+} \mu^{-} \mu^{+}$candidates that pass the final selection and are located within $3 \sigma$ of the Gaussian peaks describing the $D_{(s)}^{+}$signals. The binning used in the analysis, separating resonant and low and high non-resonant contributions, is overlaid on top of the histogram. 
The considered backgrounds are the combinatorial and the peaking $D_{(s)}^{+} \rightarrow \pi^{+} \pi^{-} \pi^{+}$decay with double $\mu \rightarrow \pi$ misidentification. A simultaneous fit to the $\pi^{+} \mu^{+} \mu^{-}$invariant mass is performed in each of the $m\left(\mu^{+} \mu^{-}\right)$bins in order to extract the non-resonant $D_{(s)}^{+} \rightarrow \pi^{+} \mu^{-} \mu^{+}$signal. No signal is observed, and therefore an upper limit is set using the $C L_{s}$ method,

$$
\begin{array}{ll}
\mathscr{B}\left(D^{+} \rightarrow \pi^{+} \mu^{-} \mu^{+}\right)<7.3(8.3) \times 10^{-8} & \text { at } 90 \%(95 \%) \text { C.L. }, \\
\mathscr{B}\left(D_{s}^{+} \rightarrow \pi^{+} \mu^{-} \mu^{+}\right)<4.1(4.8) \times 10^{-7} & \text { at } 90 \% \text { (95\%) C.L. },
\end{array}
$$

improving previous upper limits by a factor 50 , but still one order of magnitude higher than the largest NP theory predictions.

Lepton number violating (LNV) processes such as $D_{(s)}^{+} \rightarrow \pi^{-} \mu^{+} \mu^{+}$are forbidden in the SM, but could occur through lepton mixing proceeding by a non-SM particle such as the Majorana neutrino. The search for these decays is performed in a similar way as for $D_{(s)}^{+} \rightarrow \pi^{+} \mu^{-} \mu^{+}$, but data are split into equally populated $m\left(\pi^{-} \mu^{+}\right)$bins, where the Majorana neutrino mass could peak.

Following the same fitting and normalization strategy as the previously discussed $D^{+}$decays, data are found to be compatible with the background-only hypothesis, and thus upper limits are set,

$$
\begin{array}{ll}
\mathscr{B}\left(D^{+} \rightarrow \pi^{-} \mu^{+} \mu^{+}\right)<2.2(2.5) \times 10^{-8} & \text { at } 90 \% \text { (95\%) C.L. }, \\
\mathscr{B}\left(D_{s}^{+} \rightarrow \pi^{-} \mu^{+} \mu^{+}\right)<1.2(1.4) \times 10^{-7} & \text { at } 90 \% \text { (95\%) C.L. },
\end{array}
$$

improving two orders of magnitudes over previous measurements.

\section{Conclusions}

Rare $B$ and $D$ decays, specially those which proceed through FCNC, are sensitive probes to new physics effects. The results of recent searches performed at LHCb with $1.0 \mathrm{fb}^{-1}$ of $p p$ collisions collected in 2011 at center-of-mass energy of $\sqrt{s}=7 \mathrm{TeV}$ (with the addition of $1.1 \mathrm{fb}^{-1}$ of collisions collected in 2012 at $\sqrt{s}=8 \mathrm{TeV}$ in the case of $B_{s}^{0} \rightarrow \mu^{+} \mu^{-}$) have been, in all cases, compatible with the predictions of the SM. Therefore, no evidence for physics beyond the SM has been found so far.

However, LHCb has observed very rare decays never observed before, has pushed further the limits on those that have not been observed and has studied the properties of those already known with greatly improved precision, including some measurements never performed before.

Data collected in 2012, which doubles the statistics collected in 2011 and has not been presented here, will allow to obtain a deeper understanding of rare decays in the $B$ and $D$ sectors and will allow to further constrain the possible new physics contributions.

\section{References}

[1] LHCb collaboration, A. A. Alves Jr. et al., The LHCb detector at the LHC, JINST 3 (2008) S08005.

[2] R. Aaij et al., The LHCb trigger and its performance, arXiv:1211 . 3055, submitted to JINST.

[3] A. Powell et al., Particle identification at LHCb, PoS ICHEP2010 (2010) 020, LHCb-PROC-2011-008.

[4] A. J. Buras, J. Girrbach, D. Guadagnoli, and G. Isidori, On the Standard Model prediction for $\mathscr{B}\left(\boldsymbol{B}_{s, d} \rightarrow \boldsymbol{\mu}^{+} \boldsymbol{\mu}^{-}\right)$, Eur. Phys. J. C72 (2012) 2172, arXiv:1208.0934. 
[5] K. De Bruyn et al., Branching Ratio Measurements of $B_{s}$ Decays, Phys. Rev. D86 (2012) 014027 , arXiv:1204.1735.

[6] LHCb collaboration, Tagged time-dependent angular analysis of $B_{s}^{0} \rightarrow J / \psi \phi$ decays at LHCb, LHCb-CONF-2012-002.

[7] LHCb collaboration, R. Aaij et al., First evidence for the decay $B_{s}^{0} \rightarrow \mu^{+} \mu^{-}$, Phys. Rev. Lett. 110 (2013) 021801, arXiv:1211.2674.

[8] A. L. Read, Presentation of search results: the CL $L_{s}$ technique, J. Phys. G28 (2002) 2693.

[9] Particle Data Group, J. Beringer et al., Review of particle physics, Phys. Rev. D86 (2012) 010001.

[10] D. Melikhov and N. Nikitin, Rare radiative leptonic decays $B_{(d, s)} \rightarrow l^{+} l^{-} \gamma$, Phys. Rev. D70 (2004) 114028, arXiv:hep-ph/0410146.

[11] LHCb collaboration, R. Aaij et al., Search for rare $B_{(s)}^{0} \rightarrow \mu \mu \mu \mu$ decays, Phys. Rev. Lett. 110 (2013) 211801, arXiv:1303.1092.

[12] LHCb collaboration, R. Aaij et al., Measurement of the CP asymmetry in $B^{0} \rightarrow K^{* 0} \mu^{+} \mu^{-}$decays, Phys. Rev. Lett. 110 (2013) 031801, arXiv: 1210.4492.

[13] LHCb collaboration, Differential branching fraction and angular analysis of the $B^{0} \rightarrow K^{* 0} \mu^{+} \mu^{-}$ decay, LHCb-CONF-2012-008.

[14] C. Bobeth, G. Hiller, and D. van Dyk, More benefits of semileptonic rare B decays at low recoil: CP Violation, JHEP 07 (2011) 067, arXiv:1105.0376.

[15] LHCb collaboration, R. Aaij et al., Measurement of the $B^{0} \rightarrow K^{* 0} e^{+} e^{-}$branching fraction at low dilepton mass, arXiv: 1304.3035 , submitted to JHEP.

[16] J.-J. Wang, R.-M. Wang, Y.-G. Xu, and Y.-D. Yang, The Rare decays $B^{+} \rightarrow \pi^{+} l^{+} l^{-}, \rho^{+} l^{+} l^{-}$, $B^{0} \rightarrow l^{+} l^{-}$in the R-parity violating supersymmetry, Phys. Rev. D77 (2008) 014017, arXiv:0711.0321.

[17] LHCb collaboration, R. Aaij et al., First observation of the decay $B^{+} \rightarrow \pi^{+} \mu^{+} \mu^{-}$, JHEP 12 (2012) 125, arXiv: 1210.2645.

[18] S. Fajfer, N. Kosnik, and S. Prelovsek, Updated constraints on new physics in rare charm decays, Phys. Rev. D76 (2007) 074010, arXiv: 0706.1133 ; A. Paul, I. I. Bigi, and S. Recksiegel, On $D \rightarrow X_{u} l^{+} l^{-}$within the Standard Model and Frameworks like the Littlest Higgs Model with T Parity, Phys. Rev. D83 (2011) 114006, arXiv:1101.6053; M. Artuso et al., B, D and K decays, Eur. Phys. J. C57 (2008) 309, arXiv: 0801.1833.

[19] LHCb collaboration, R. Aaij et al., A search for $D_{(s)}^{+} \rightarrow \pi^{+} \mu^{-} \mu^{-}$and $D_{(s)}^{+} \rightarrow \pi^{-} \mu^{+} \mu^{+}$decays, arXiv:1304.6365, submitted to Phys. Lett. B. 